\title{
MicroRNA-29a Counteracts Glucocorticoid Induction of Bone Loss through Repressing TNFSF13b Modulation of Osteoclastogenesis
}

\author{
Re-Wen Wu ${ }^{1, \dagger}$, Wei-Shiung Lian ${ }^{2,3, \dagger}$, Yu-Shan Chen ${ }^{2,3}$, Chung-Wen Kuo ${ }^{2,3}$, Huei-Ching Ke ${ }^{2,3}$, \\ Chin-Kuei Hsieh ${ }^{2,3}$, Shao-Yu Wang ${ }^{2,3}$, Jih-Yang Ko ${ }^{1, *}$ and Feng-Sheng Wang ${ }^{2,3,4, *}$ \\ 1 Department of Orthopedic Surgery, Kaohsiung Chang Gung Memorial Hospital, Kaohsiung 83301, Taiwan; \\ ray4595@gmail.com \\ 2 Core Laboratory for Phenomics and Diagnostic, Kaohisung Chang Gung Memorial Hospital, \\ Kaohsiung 83301, Taiwan; lianws@gmail.com (W.-S.L.); ggyy58720240@gmail.com (Y.-S.C.); \\ bulakuo@gmail.com (C.-W.K.); maggie2258@cgmh.org.tw (H.-C.K.); jorno0329@gmail.com (C.-K.H.); \\ vip690221@gmail.com (S.-Y.W) \\ 3 Department of Medical Research, Kaohisung Chang Gung Memorial Hospital, Kaohsiung 83301, Taiwan \\ 4 Graduate Institute of Clinical Medical Sciences, Chang Gung University College of Medicine, \\ Kaohsiung 83301, Taiwan \\ * Correspondence: kojy@cgmh.org.tw (J.-Y.K.); wangfs@ms33.hinet.net (F.-S.W.); \\ Tel.: +886-7-731-7123 (ext. 6406) (F.-S.W.) \\ + These authors contributed equally to this work.
}

Received: 12 September 2019; Accepted: 14 October 2019; Published: 17 October 2019

\begin{abstract}
Glucocorticoid excess escalates osteoclastic resorption, accelerating bone mass loss and microarchitecture damage, which ramps up osteoporosis development. MicroRNA-29a (miR-29a) regulates osteoblast and chondrocyte function; however, the action of miR-29a to osteoclastic activity in the glucocorticoid-induced osteoporotic bone remains elusive. In this study, we showed that transgenic mice overexpressing an miR-29a precursor driven by phosphoglycerate kinase exhibited a minor response to glucocorticoid-mediated bone mineral density loss, cortical bone porosity and overproduction of serum resorption markers C-teleopeptide of type I collagen and tartrate-resistant acid phosphatase $5 \mathrm{~b}$ levels. miR-29a overexpression compromised trabecular bone erosion and excessive osteoclast number histopathology in glucocorticoid-treated skeletal tissue. Ex vivo, the glucocorticoid-provoked osteoblast formation and osteoclastogenic markers (NFATc1, MMP9, V-ATPase, carbonic anhydrase II and cathepsin K) along with F-actin ring development and pit formation of primary bone-marrow macrophages were downregulated in miR-29a transgenic mice. Mechanistically, tumor necrosis factor superfamily member 13b (TNFSF13b) participated in the glucocorticoid-induced osteoclast formation. miR-29a decreased the suppressor of cytokine signaling 2 (SOCS2) enrichment in the TNFSF13b promoter and downregulated the cytokine production. In vitro, forced miR-29a expression and SOCS2 knockdown attenuated the glucocorticoid-induced TNFSF13b expression in osteoblasts. miR-29a wards off glucocorticoid-mediated excessive bone resorption by repressing the TNFSF13b modulation of osteoclastic activity. This study sheds new light onto the immune-regulatory actions of miR-29a protection against glucocorticoid-mediated osteoporosis.
\end{abstract}

Keywords: miR-29a; osteoclasts; bone loss; TNFSF13b

\section{Introduction}

Glucocorticoid overmedication dysregulates bone cell activity, accelerating bone mass loss and structure deterioration, which increases the development of osteoporotic diseases [1]. Osteoclast 
overdevelopment that progressively erodes bone microstructure [2] and weakens biomechanical properties of skeletal tissues [3] is a notable pathological feature of glucocorticoid-induced osteoporosis. Increasing studies have shown the involvement of intracellular pathways, like vanilloid receptor, cannabinoid receptor [4] and circadian rhythm [5], in glucocorticoid-mediated osteoclast activation.

On the other hand, glucocorticoids also facilitate osteogenic cells and bone marrow cells to produce osteoclastogenesis-promoting factors, like tumor necrosis factor superfamily (TNFSF) member receptor activator NF- $\mathrm{KB}$ ligand (RANKL), which is indispensable in osteoclast differentiation and activation [6]. Several TNFSF members are also involved in the RANKL-independent osteoclast differentiation program. Of them, CD137 promotes osteoclast migration and differentiation in breast cancer-induced bone metastasis [7]. Mice deficient in TNFSF14 show increased bone mass together with suppressed osteoclastic resorption [8]. TNFSF13b promotes the shifting of human monocytes into osteoclastic cells [9], whereas blocking TNFSF13b reverses multiple myeloma-mediated osteoclast formation [10].

MicroRNA belong to non-coding small RNA, disrupting mRNA targets and regulating tissue development, remodeling and malignancy [11]. Many microRNAs alter osteoclast survival, differentiation and maturation under osteoporotic and arthritic conditions. For example, miR-27a mediates the estrogen-induced loss of osteoclast differentiation and bone resorption capacity of bone-marrow macrophages [12]. miR-31a-5p accelerates osteoclast formation in an age-mediated osteoporotic skeleton [13]. miR-124 downregulates osteoclastic resorption in arthritic joints in rats upon adjuvant injection [14]. miR-34 inhibits osteoclast activation in the development of osteoporosis and osteolytic bone metastasis [15]. Moreover, the miR-29 family regulates myogenesis of muscle stem cells [16], chondrogenic differentiation of mesenchymal stem cells [17] and fibrosis matrix formation in inflamed tissue [18]. miR-29 signaling also modulates immune cell activation in multiple sclerosis [19], the host immune response [20] and inflammatory reaction in colitis [21]. We previously revealed that glucocorticoid induced bone loss and marrow adipose overdevelopment along with decreased miR-29a expression [22]. The biological function of miR-29a in osteoclast behavior in glucocorticoid-treated bone tissue is not well understood.

This study aimed to investigate whether osteoclast differentiation or bone resorption in the glucocorticoid-induced osteoporotic skeleton was changed in miR-29a transgenic mice (miR-29aTg) and tested whether cytokine TNFSF13b mediated the miR-29a regulation of glucocorticoid-provoked osteoclast formation.

\section{Results}

\section{1. miR-29 Overexpression Compromised Glucocorticoid-Induced Bone Loss}

We utilized miR-29aTg mice to test whether increasing miR-29a transcripts in trabecular bone, as evident from in situ hybridization (Figure 1A), altered bone mass or osteoclastic resorption in skeletal tissue upon glucocorticoid treatment. Bone resorption markers C-teleopeptide of type I collagen (CTX-1) and tartrate-resistant acid phosphatase 5b (TRAP5b) levels in sera were significantly increased in wild-type (WT) mice upon $5 \mathrm{mg} / \mathrm{kg} /$ day methylprednisolone treatment for 28 days, whereas they were significantly downregulated in glucocorticoid-treated miR-29aTg mice (Tg) (Figure 1B). Of note, glucocorticoid-treated WT mice exhibited severely poor trabecular bone microstructure (Figure 1C) along with significantly decreased bone mineral density and increased cortical bone porosity (Figure 1D). Well-woven trabecular bone architecture together with minor bone loss and porosity occurred in glucocorticoid-treated miR-29aTg mice. 
A

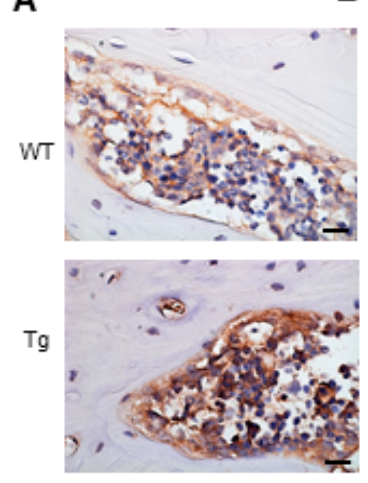

B

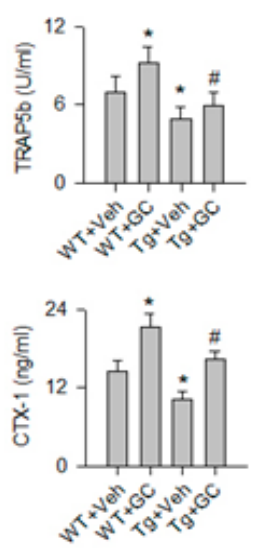

C

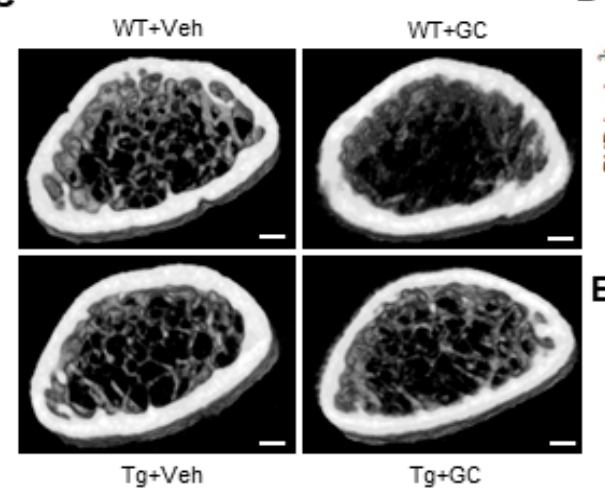

D

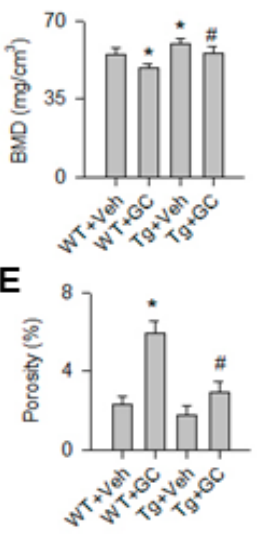

Figure 1. Analysis of bone mass, microstructure and resorption markers in bone tissue in WT mice and miR-29aTg mice. Strong miR-29a transcripts in miR-29aTg bone tissue (A); scale bar, $10 \mu \mathrm{m}$. miR-29a overexpression downregulated serum TRAP5b and CTX-1 levels (B). Glucocorticoid-treated WT mice showed sparse trabecular bone, whereas abundant trabecular microstructure remained in glucocorticoid-treated miR-29aTg mice (C); scale bar, $5 \mathrm{~mm}$. miR-29a overexpression improved bone mineral density (D) and cortical bone porosity (E) in glucocorticoid-treated skeleton. Data are expressed as the mean \pm standard errors calculated from 6 mice. Asterisks * indicate significant differences from the WT + Veh group and hashtags \# indicate significant differences from the WT + GC group $(p<0.05)$. WT, wild-type mice; Tg, miR-29aTg mice; Veh, vehicle; GC, glucocorticoid. TRAP5b, tartrate-resistant acid phosphatase 5b; CTX-1, C-telopeptide of type I collagen; BMD, bone mineral density.

\section{2. miR-29 Repressed the Glucocorticoid-Induced Osteoclastic Erosion Histopathology}

In addition, bone tissue in glucocorticoid-treated WT mice showed severe trabecular loss and increased osteoclast formation histopathology as evident from TRAP (tartrate-resistant acid phosphatase) staining, whereas specimens from glucocorticoid-treated miR-29aTg mice displayed abundant trabecular bone together with mild osteoclast distribution (Figure 2A). Consistently, glucocorticoid significantly increased trabecular separation (Tb.Sp; Figure 2B), osteoclast number (Oc.N; Figure 2C), erosion area (Figure 2D) and eroded surface (ES.BS\%; Figure 2E) in WT mice. miR-29a overexpression reversed the bone resorption histomorphology in glucocorticoid-treated skeleton.

\section{3. miR-29a Inhibited Osteoclast Differentiation and Resorption Capacity}

The miR-29a improvement of bone erosion in glucocorticoid-treated bone tissue prompted us to isolate primary bone-marrow macrophages for characterizing osteoclast activity in WT mice and miR-29a mice. Numerous enlarged osteoclasts positive for TRAP staining formed in glucocorticoid-treated WT mice; these phenomena were improved in the glucocorticoid-treated miR-29aTg group (Figure 3A). Glucocorticoid significantly increased osteoclast number and area (Figure 3B) and also upregulated osteoclastogenic markers NFATc1, cathepsin K (Figure 3C), mature osteoclast markers carbonic anhydrase II and vacuolar $\mathrm{H}^{+}$-ATPase expression (Figure 3D) in the WT group. miR-29a overexpression significantly downregulated osteoclast formation and osteoclast marker expression of bone-marrow macrophages below the baseline and also improved the glucocorticoid-upregulated osteoclast differentiation. 
A

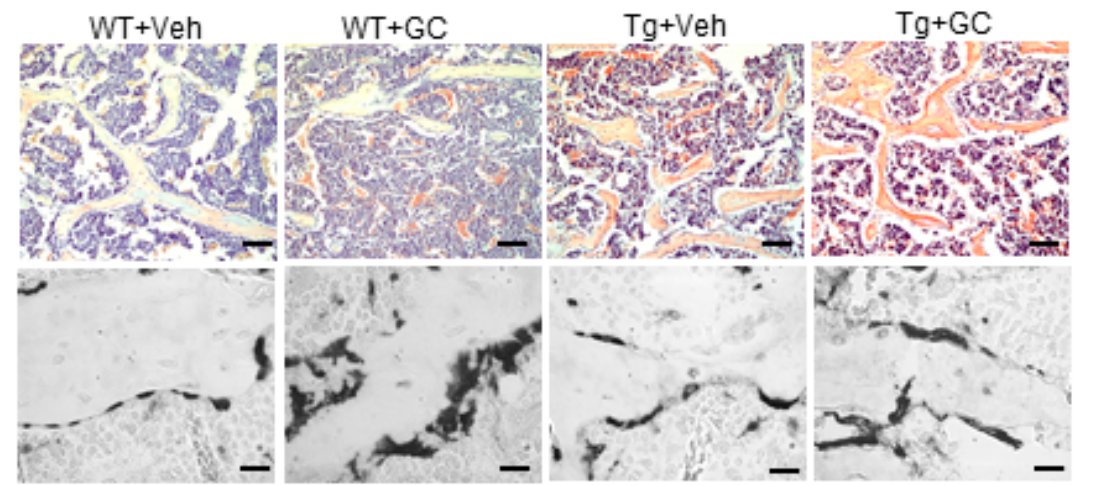

B

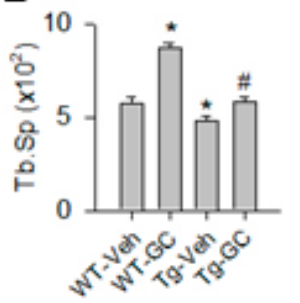

C

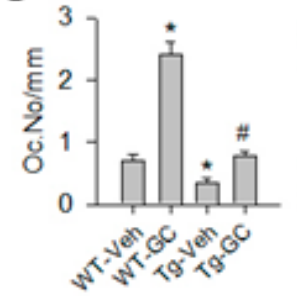

D

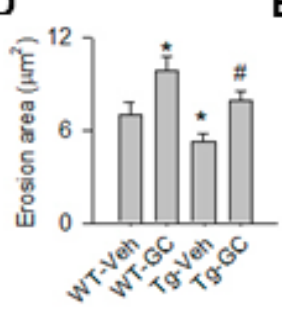

E

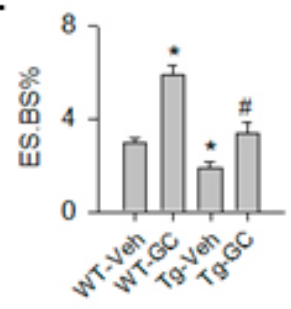

Figure 2. Histological analysis of trabecular bone and osteoclast distribution. Severe trabecular bone loss and increased TRAP-stained osteoclasts existed in glucocorticoid-treated WT bone tissue, whereas well-connected bone histology but few osteoclasts remained in glucocorticoid-treated miR-29aTg bone tissue (A); Scale bar, $30 \mu \mathrm{m}$ (upper panels); $10 \mu \mathrm{m}$ (lower panel). The glucocorticoid-mediated increases in Tb.Sp (B), Oc.N (C), erosion area (D) and ES.BS\% (E) were significantly improved in miR-29aTg mice. Data are expressed as the mean \pm standard errors calculated from 6 mice. Asterisks * indicate significant differences from the WT-Veh group and hashtags \# indicate significant differences from the WT-GC group $(p<0.05)$. WT, wild-type mice; Tg, miR-29aTg mice; Veh, vehicle; GC, glucocorticoid; $\mathrm{Tb} . \mathrm{Sp}$, trabecular separation; Oc.N, osteoclast number; ES.BS, eroded surface.

B

A

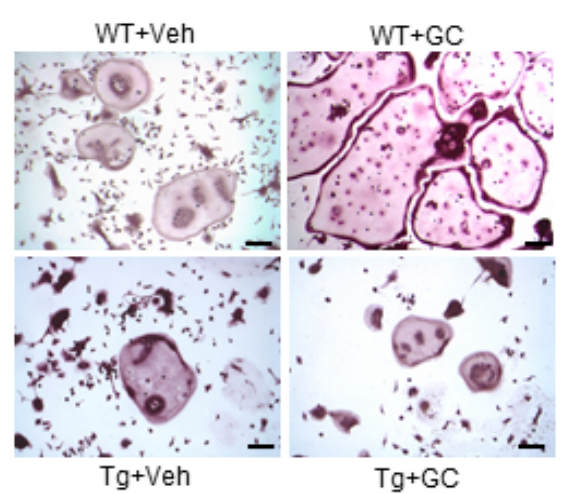

C

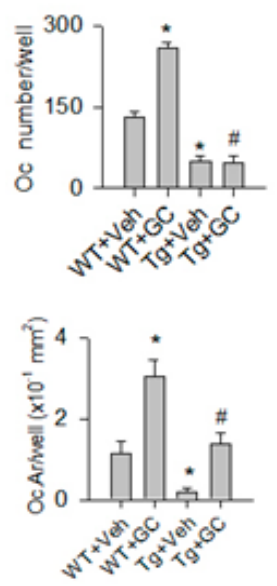

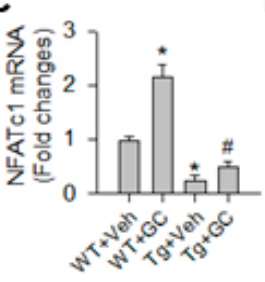

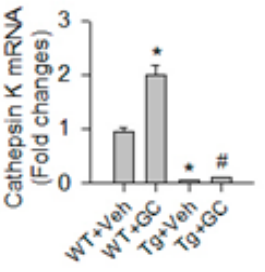

D
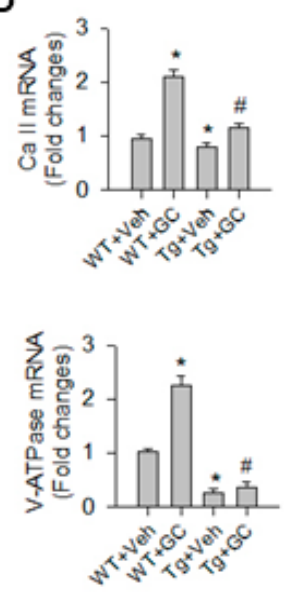

Figure 3. Analysis of osteoclast differentiation of primary bone-marrow macrophages. Increased and enlarged osteoclasts positive for TRAP staining occurred in glucocorticoid-treated WT mice, whereas few osteoclasts formed in miR-29aTg mice (A) scale bar, $8 \mu \mathrm{m}$. miR-29a overexpression repressed the glucocorticoid-induced increases in osteoclast number and area (B) and also reduced osteoclastogenic markers NFATc1, cathepsin K (C), and osteoclast maturation markers carbonic anhydrase II and V-ATPase expression (D). Data are expressed as the mean \pm standard errors calculated from 6 mice. Asterisks * indicate significant differences from the WT + Veh group and hashtags \# indicate significant differences from the WT + Veh group $(p<0.05)$. WT, wild-type mice; Tg, miR-29aTg mice; Veh, vehicle; GC, glucocorticoid, Oc, osteoclasts; Oc.Ar, osteoclast area; NFATc1, nuclear factor of activated T-cells-c1; Ca II, carbonic anhydrase II; V-ATPase, vacuolar $\mathrm{H}^{+}$-ATPase. 
In addition, osteoclasts in glucocorticoid-treated wild type (WT) mice showed strongly fluorescent F-actin ring morphology (Figure 4A) along with significant increases in F-actin rings (Figure 4B) and matrix metallopeptidase 9 (MMP9) expression (Figure 4C). miR-29a overexpression significantly repressed these reactions in osteoclasts from glucocorticoid-treated skeleton. Moreover, osteoclast precursor cells were incubated onto the bone biomimetic surface to characterize pit formation (Figure 4D). Osteoclasts from glucocorticoid-treated WT mice eroded larger area of pits as compared with vehicle-treated WT mice. This activity was significantly downregulated in glucocorticoid-treated miR-29aTg mice (Figure 4E). Gain of miR-29 signaling significantly reduced osteoclastic resorption capacity below the baseline.
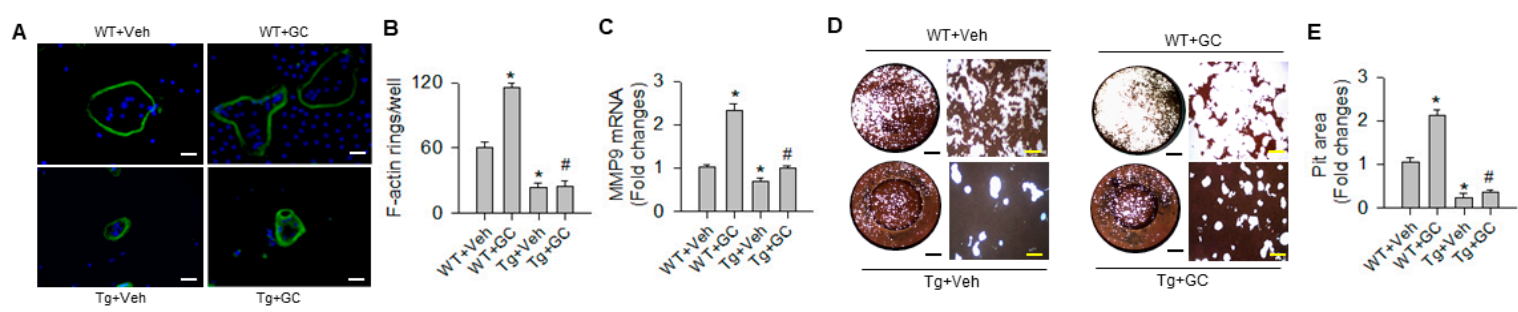

Figure 4. Analysis of F-actin ring formation and pit formation of bone-marrow osteoclastogenic cells. Osteoclasts in glucocorticoid-treated WT mice showed strongly fluorescent F-actin ring morphology (A) (scale bar, $20 \mu \mathrm{m}$ ) and increases in F-actin ring number (B), MMP9 expression (C) and pit formation (D, E); (black scale bar, $7 \mathrm{~mm}$; yellow scale bar, $30 \mu \mathrm{m}$ ). These effects were compromised in glucocorticoid-treated miR-29aTg mice. Data are expressed as the mean \pm standard errors calculated from 6 mice. Asterisks * indicate significant differences from the WT + Veh group and hashtags \# indicate significant differences from the WT + GC group $(p<0.05)$. WT, wild-type mice; Tg, miR-29aTg mice; Veh, vehicle; GC, glucocorticoid; MMP9, matrix metalloproteinase 9.

\subsection{TNFSF13b-Mediated miR-29a Regulation of Osoteoclast Formation}

Bioinformatics reveal that TNFSF13b is one of putative targets of miR-29 (www.microrna.org). This cytokine is shown to promote monocyte differentiation toward osteoclastic lineages $[9,10]$. Of note, osteoblasts adjacent to trabecular bone in glucocorticoid-treated WT mice exhibited strong TNFSF13b immunostaining, whereas bone cells in glucocorticoid-treated bone tissue displayed weak TNFSF13b immunoreactivity (Figure 5A). Consistent with the histomorphometric analysis (Figure 5B), serum TNFSF13b levels were significantly increased in WT mice upon glucocorticoid treatment. miR-29a overexpression attenuated the glucocorticoid-augmented TNFSF13b levels (Figure 5C).

We tested whether TNFSF13b participated in the miR-29a reduction of osteoclastogenic activities in glucocorticoid-treated skeleton. TNFSF13b antibody or TNFSF13b protein were added to bone-marrow macrophages from WT mice and miR-29aTg mice with glucocorticoid treatment. Of interest, blocking TNFSF13b significantly decreased osteoclast formation of macrophage cultures from glucocorticoid-treated WT mice (Figure 5D). On the contrary, co-incubation with TNFSF13b protein significantly increased osteoclast differentiation of bone-marrow macrophages from glucocorticoid-treated miR-29aTg mice (Figure 5D), which is suggestive of the involvement of TNFSF13b in miR-29a regulation of osteoclast differentiation.

\subsection{SOCS2 Controlled the miR-29a Inhibition of TNFSF13b Signaling}

Bioinformatics searches (www.cbil.upenn/cgi-bin/tess/tess) revealed that SOCS2 is a putative transcription factor for TNFSF13b transcription. miR-29a overexpression significantly attenuated the glucocorticoid-induced increases in SOCS2 levels in bone tissue (Figure 6A). In addition, glucocorticoid significantly increased the SOCS2 enrichment in the TNFSF13b promoter as evident from chromatin immunoprecipitation (ChIP)-PCR analysis (Figure 6B) along with a significant increase in TNFSF13b mRNA transcription (Figure $6 \mathrm{C}$ ) in WT bone tissue. These effects were significantly compromised in miR-29aTg skeletal tissue. SOCS2 monoclonal antibodies used in ChIP experiments exhibited 
prominent enrichment in the TNFSF13b proximal promoter region as compared to IgG, which is suggestive of high specificity to the promoter region of interest.

A

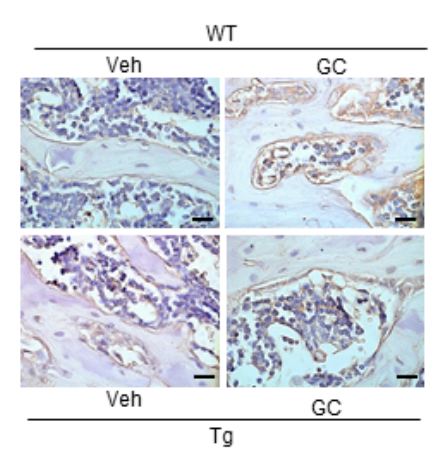

B

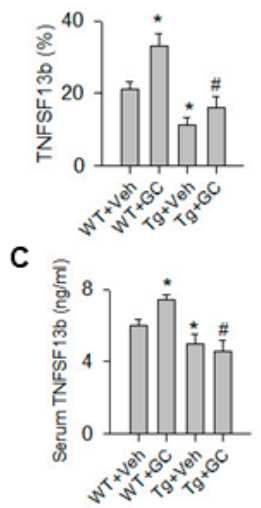

D

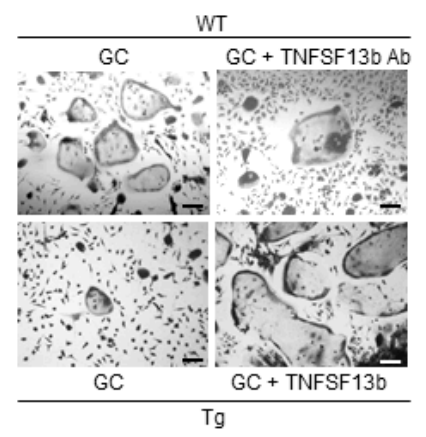

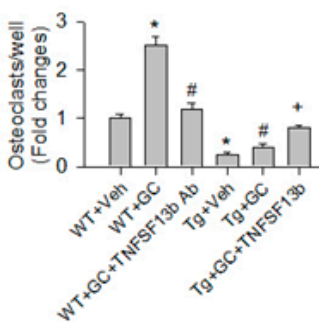

Figure 5. Analysis of TNFSF13b immunohistochemistry and action in osteoclast formation. Bone cells in glucocorticoid-treated WT mice showed strong TNFSF13b immunostaining, but expressed weak immunoreaction in glucocorticoid-treated miR-29aTg mice (A); scale bar, $10 \mu \mathrm{m}$. miR-29a overexpression attenuated the glucocorticoid-induced increases in TNFSF13b immunostaining (B) and serum TNFSF13b levels (C). TNFSF13b antibody blockade attenuated the glucocorticoid-induced osteoclast formation of bone-marrow macrophages from WT mice, whereas TNFSF13b protein increased osteoclast formation of bone-marrow macrophages from glucocorticoid-treated miR-29aTg mice (D); scale bar, $8 \mu \mathrm{m}$. Data are expressed as the mean \pm standard errors calculated from 6 mice. Asterisks * indicate significant differences from the WT + Veh group, hashtags \# indicate significant differences from the WT + GC group and plus + indicate significant difference the Tg + GC group $(p<0.05)$. WT, wild-type mice; Tg, miR-29aTg mice; Veh, vehicle; GC, glucocorticoid; TNFSF13b, tumor necrosis factor superfamily $13 \mathrm{~b} ; \mathrm{Ab}$, antibody.

A
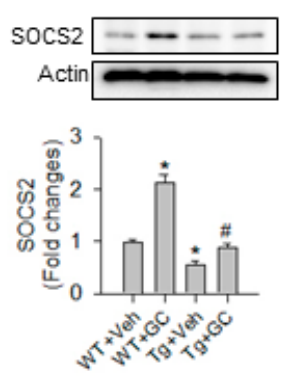

B
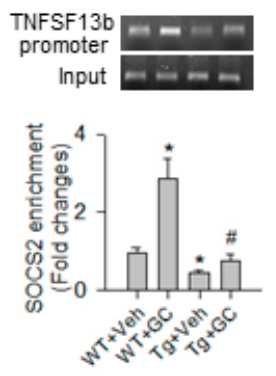

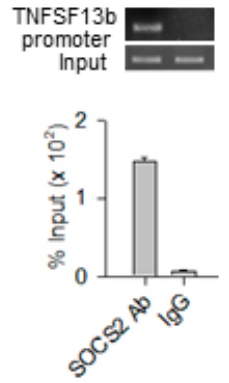

C

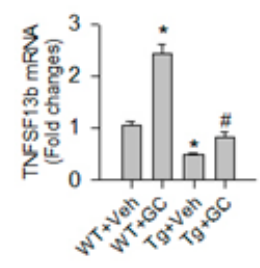

Figure 6. Analysis of SOCS2 immunoblotting and ChIP-PCR of SOCS2 enrichment in TNFSF13b. miR-29a overexpression attenuated the glucocorticoid-induced increase in SOCS2 protein (A), the SOCS2 enrichment in the TNFSF13b promoter region (B), and TNFSF13b mRNA expression (C). Data are expressed as the mean \pm standard errors calculated from 3-6 mice. Asterisks * indicate significant differences from the WT + Veh group and hashtags \# indicate significant differences from the WT + GC group $(p<0.05)$. Veh, vehicle; GC, glucocorticoid; SOCS2, suppressor of cytokine signaling 2; $\mathrm{Ab}$, antibody.

Consistent with the analysis of the in vivo model, glucocorticoid and miR-29a knockdown significantly decreased miR-29a expression in osteoblast cultures (Figure 7A). Gain of miR-29a signaling significantly attenuated the glucocorticoid-provoked TNFSF13b expression (Figure 7B). Moreover, glucocorticoid significantly increased SOCS2 expression. Loss of SOCS2 function (Figure 7C) downregulated TNFSF13b expression in osteoblast cultures upon glucocorticoid stress (Figure 7D). 
A

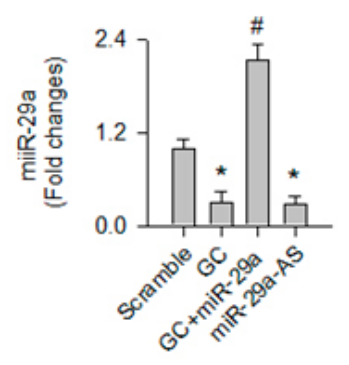

B

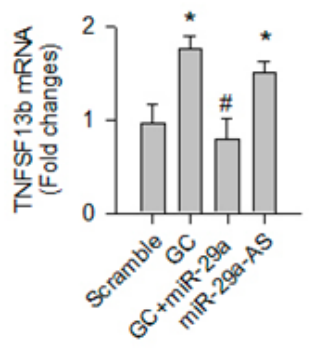

C

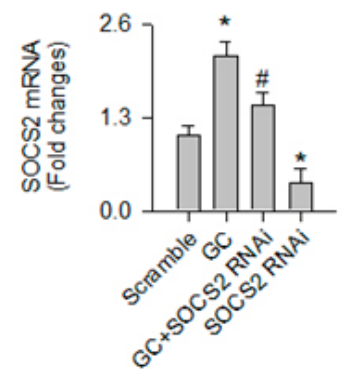

D

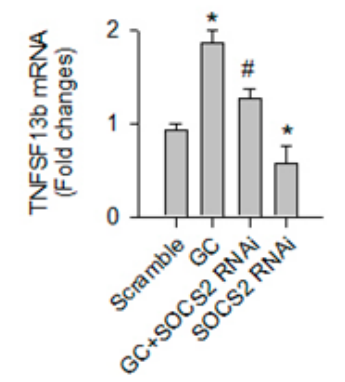

Figure 7. Analysis of TNFSF13b and SOCS2 expression in osteoblasts. Forced miR-29a expression (A) attenuated the glucocorticoid-provoked TNFSF13b expression in osteoblasts, whereas loss of miR-29a function increased the cytokine expression (B). SOCS2 knockdown (C) attenuated the glucocorticoid-mediated increases in TNFSF13b expression (D). Data are expressed as the mean \pm standard errors calculated from 3 experiments. Asterisks * indicate significant differences from the scramble group and hashtags \# indicate significant differences from the GC group. GC, glucocorticoid; miR-29a-AS, miR-29a antisense oligonucleotide; RNAi, RNA interference.

\section{Discussion}

Physiological levels of glucocorticoids are essential to maintain the contact of osteoclastic cells with the mineralized matrix, harmonizing the bone turnover reaction beneficial for bone mass homeostasis [6,23]. Glucocorticoid excess increases osteoclast survival [24] and resorption activity [25], overwhelmingly shattering bone mass and structure. While accumulating evidence has revealed the microRNA signaling regulation of osteoclast differentiation and bone erosion in degenerative and metastatic conditions [26,27], little is known of the role microRNAs may play in osteoclast overgrowth in the development of glucocorticoid-induced osteoporosis. Collective analysis in this study revealed that miR-29a downregulated the glucocorticoid-augmented osteoclastic resorption, fending off osteoporotic skeleton development. Decreased cytokine TNFSF13b signaling in bone microenvironment contributed to the miR-29a repression of osteoclast function. This study offers a new epigenetic insight into how the microRNA pathway controls osteoclast behavior, delaying the development of glucocorticoid-induced bone loss. Robust analysis is also the first indication to highlight the contribution of TNFSF13b signaling to this osteoporotic disorder.

In this study, treatment with a high dose of methylprednisolone deteriorated bone mass along with significant increases in skeletal porosity and serum bone resorption markers, like TRAP5b and CTX-1. Our investigations are in agreement with other groups' studies showing that glucocorticoid administration increased serum TRAP5b levels [28] and cortical bone loss [29]. Furthermore, miR-29a overexpression attenuated the glucocorticoid-induced osteoblast dysfunction and marrow adipose deposition [22], which is indicative that miR-29a signaling may alter osteoclast behavior in glucocorticoid-treated bone tissue. These findings reasoned us to employ miR-29aTg mice to understand whether miR-29a affected the osteoclastic resorption reaction in the glucocorticoid excess-stressed bone microenvironment.

Of note, a plethora of glucocorticoid-induced excessive bone resorption signs, like resorption marker overproduction, bone cortical porosity and trabecular bone erosion histopathology, were significantly downregulated in miR-29aTg mice, which is suggestive of miR-29a signaling suppression of bone remodeling in glucocorticoid-treated skeletal tissue. The analysis of decreased osteoclast distribution in miR-29aTg bone tissue underpinned the investigations of its protective effects on bone mass homeostasis. In addition, miR-29a overexpression reversed the glucocorticoid upregulation of osteoclast differentiation capacity, like osteoclast formation, maturation and pit formation. The investigations of osteoclast differentiation of bone-marrow macrophages were consistent with the in vivo findings. However, the effect of the miR-29 family members on osteoclastogenic lineage specification or osteoclast maturation remains uncertain. For example, knocking down miR-29a, 
b or c decreases osteoclast differentiation capacity of RAW264.7 monocytic cells, whereas survival and F-actin ring formation were not significantly affected in mature osteoclasts [30]. Treatment with miR-29a mimic promotes osteoclast survival rather than differentiation; however, the miR-29a inhibitor downregulates lipopolysaccharide-induced osteoclast growth [31]. On the contrary, forced miR-29b expression inhibits osteoclast markers nuclear factor of activated T-cell, cytoplasmic 1 (NFATc1) and matrix metallopep (MMP9) expression and also reduces collagen degradation and pit formation of osteoclastic cultures in a multiple myeloma-mediated osteolysis model [32]. This study uncovered that miR-29a overexpression suppressed osteoclast formation in glucocorticoid excess-treated skeleton. We speculated that the miR-29 action to osteoclast formation may depend on osteoporotic disease and osteoclastogenic progenitor cell types. The repressed osteoclastic resorption in glucocorticoid-treated miR-29aTg bone tissue further explains the complex nature of glucocorticoid-induced osteoporosis.

The miR-29a hinderance of osteoclast activity promoted us to pinpoint what cytokine contributed to this reaction. TNFSF members, like RANKL, TNFSF13b and TNFSF14, etc., regulate differentiation, maturation and activation of osteoclastogenic cells in the development of osteoporosis caused by estrogen loss, arthritis or bone metastasis [33,34]. A decrease in RANKL rather than osteoprotegerin (OPG) expression occurs in the miR-29a regulation of glucocorticoid excess-treated skeletons [22]. TNFSF13b is shown to promote osteoclast differentiation of human monocytic cultures in the absence of RANKL [9]. Of note, this study uncovered the involvement of TNFSF13b in the glucocorticoid-provoked osteoclast activities, as significant increases in TNFSF13b secretion and immunostaining occurred in glucocorticoid-treated mice. TNFSF13b antibody blockade mitigated the glucocorticoid upregulation of osteoclast formation of bone-marrow macrophages. Adding TNFSF13b protein to miR-29aTg bone-marrow macrophages weakened the miR-29a inhibition of osteoclast formation. Intriguing analysis sheds a new light on the TNFSF action in the development of glucocorticoid-induced bone damage.

Furthermore, SOCS2 is a master transcription factor regulating polarization and lineage commitment of macrophages [35]. Mice deficient in SOCS2 show increased bone growth and widened growth plate [36] and display an amplified response to growth hormone promotion of bone development [37]. This immune regulator is a putative transcription factor for TNFSF13b transcription (www.cbil.upenn/cgi-bin/tess/tess). Collective analysis confirmed that miR-29a overexpression attenuated the glucocorticoid upregulation of SOCS2 levels and the SOCS2 occupancy in TNFSF13b promoter, which increased TNFSF13b expression. In vitro, knockdown of SOCS2 downregulated the glucocorticoid-mediated TNFSF13b expression in osteoblasts. The miR-29 family members are emerging immune regulators for immune disorders [18,19], atherosclerotic diseases [38], and malignant tumor formation $[39,40]$. This study reveals a new immune-regulatory mechanistic by which miR-29a delays osteoclastic resorption in glucocorticoid-treated bone tissue. We do not exclude the possibility that other TNFSF members may be involved in the miR-29a protection from glucocorticoid aggravation of osteoclastic resorption and bone loss. Serine/threonine kinase Pim together with NFkB pathways is shown to regulate TNFSF13b-mediated survival of multiple myeloma cells and osteoclasts [41]. The molecular events underlying TNFSF13b modulation of glucocorticoid-mediated osteoclast formation and the effect of a TNFSF13b inhibitor, like Belimumab, on the glucocorticoid-induced excessive osteoclastic resorption warrants investigation in the future.

Taken together, miR-29 signaling represses the glucocorticoid-induced excessive osteoclast formation and bone resorption, slowing the development of osteoporotic skeleton. miR-29a improves the glucocorticoid-induced osteoclast overdevelopment by reducing SOCS2 and TNFSF13b signaling (Figure 8). Profound analyses convey a new epigenetic insight into microRNA shielding from glucocorticoid-induced excessive bone remodeling and bone loss. 


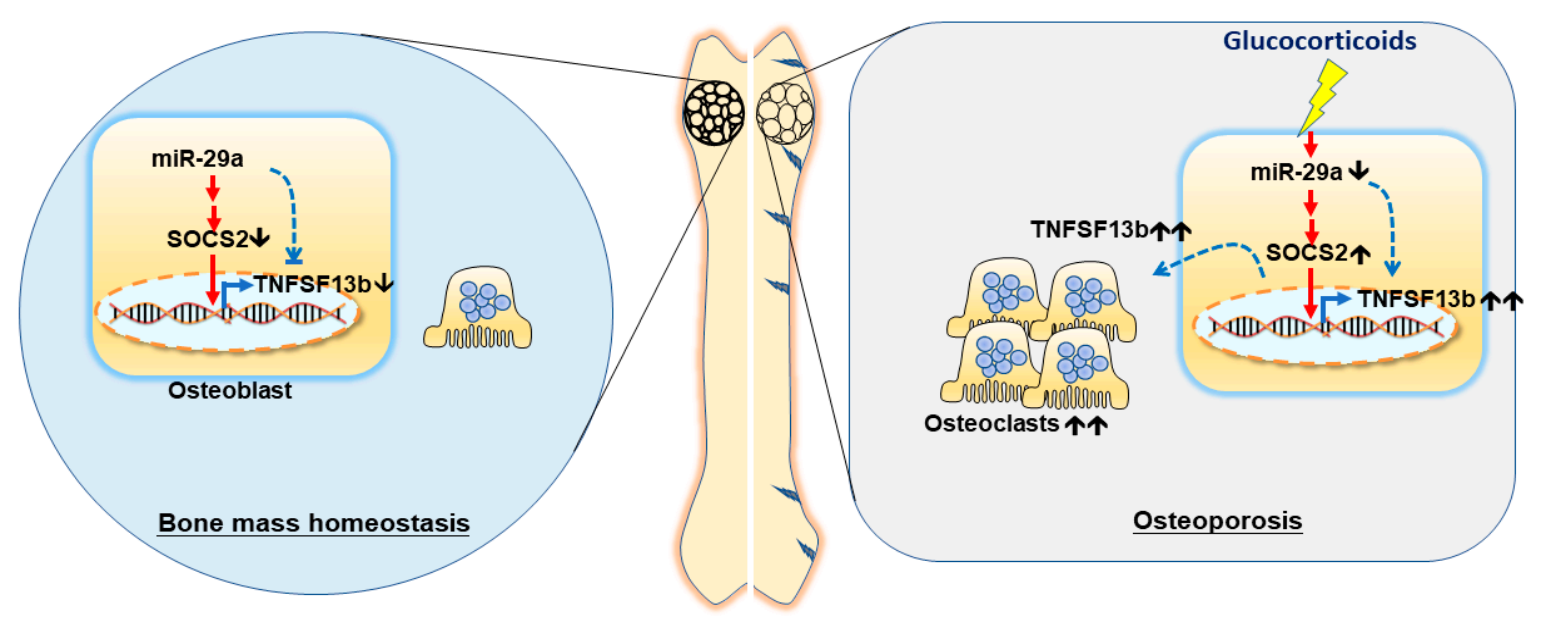

Figure 8. miR-29a attenuates the glucocorticoid-induced TNFSF13b overproduction, improving excessive osteoclast formation in the development of glucocorticoid-mediated osteoporosis. $\uparrow$, upregulation; $\uparrow$, downregulation; and $\uparrow \uparrow$, overexpression. Blue dotted line, a direct action; red solid line, an indirect effect.

\section{Materials and Methods}

\section{1. miR-29a Transgenic Mice}

Protocols for animal breeding, experimentation and care were reviewed and approved by the Institutional Animal Use and Care Committee in March, 2011, Kaohsiung Chang Gung Memorial Hospital in September, 2012 (Affidavit No. 2011030701 and No.2012091003). (Friend leukemia virus B; FVB) mice overexpressing miR-29a precursor (FVB/TNar-Tg-29a/PGK; miR-29aTg) driven by the phosphoglycerate kinase (PGK) promoter were bred, as previously described [22]. The genotype of each animal was confirmed using customized primers (forward: 5'-GAGGATCCCCTCAAGGATACCAAGGGATGAAT-3'; reverse, 5'-CTTCTAGAAGGAGTGTTTCTAGGTATCCGTCA-3') along with PCR analysis [22].

\subsection{Glucocorticoid-Induced Osteoporosis}

Male wild-type mice (WT) and miR-29aTg mice (Tg; 12 weeks old) were intraperitoneally given $5 \mathrm{mg} / \mathrm{kg} /$ day methylprednisolone or vehicle for 28 days. Upon euthanasia, peripheral blood was harvested via an intra-cardiac puncture, and tibia and femurs were dissected for $\mu \mathrm{CT}$ and histological assessment.

\subsection{Quantification of Serum Bone Resorption Markers}

Designated ELISA kits were utilized to quantify tartrate-resistant acid phosphate $5 b$ (TRAP5b; Biomedical Technologies Inc., Stoughton, MA, USA), C-teleopeptide of type I collagen (CTX-I; Nordic Bioscience Diagnostics, Herlev, Denmark) and TNFSF13b (R \& D Systems, Minneapolis, MN, USA) levels in sera, according to the manufacturers' manuals.

\subsection{Assay of Bone Mass and Microstructure}

$\mu \mathrm{CT}$ analysis of bone mineral density, trabecular microstructure and cortical bone porosity was performed using a Skyscan $1176 \mu \mathrm{CT}$ system (Bruker, Kontich, Belgium). Specimens were subjected to $50-\mathrm{kev}, 500-\mu \mathrm{A}$, and $69-\mathrm{ms}$ radiography and followed by reconstructing 200 slices of radiographs (isotropic 9- $\mu \mathrm{m}$ voxel each slice) into 3D images using SKYSCAN ${ }^{\circledR}$ CT-Analyzer software (Bruker, Kontich, Belgium). Trabecular bone mineral density $\left(\mathrm{mg} / \mathrm{cm}^{3}\right)$ and cortical bone porosity $(\%)$ were quantified according to the manufacturer's instructions. 


\subsection{In Situ Hybridization and Histomorphometry}

Customized miR-29a probes conjugated with digoxigenin (Applied Biosystems, Carlsbad, CA, USA) along with IsHyb In Situ Hybridization kits (Biochain Institute, Inc., Newark, CA, USA) were utilized to probe miR-29a transcripts in bone tissue, as previously described [22]. Specimens were subjected to hematoxylin and eosin staining and tartrate-resistant acid phosphatase histochemical staining. Osteoclast morphology was microscopically evaluated, as previously described [42]. Three fields in each section and 12 sections from 6 mice were randomly selected for histomorphometry using a Ziess microscope (ZIESS, Munchen, Germany) and Image-Pro ${ }^{\circledR}$ Plus image-analysis software (Media Cybernetics Inc., Rockville, MD, USA). Trabecular separation (\%), osteoclast number (Oc.No/mm), erosion area $\left(\mu \mathrm{m}^{2}\right)$, and erosion surface (ES.BS\%) were calculated [43].

\subsection{Ex Vivo Osteoclast Differentiation and F-Actin Ring Immunofluorescence Labeling}

Primary bone-marrow macrophages in tibiae and femurs were isolated, as previously described [44]. In brief, nucleated cells in bone marrow were isolated upon lysis of red blood cells using RBC Lysis buffer (Sigma-Aldrich Co., St Louis, MO, USA) and incubated in $\alpha$-MEM with $10 \%$ fetal bovine serum and $20 \mathrm{ng} / \mathrm{mL}$ M-CSF (R\&D Systems, Minneapolis, MN, USA) for 1 day. The floating cells were harvested upon incubation. Then, $10^{5} /$ well macrophages (24-well plates) were incubated in osteoclastogenic medium containing $\alpha$-MEM, 10\% fetal bovine serum, $20 \mathrm{ng} / \mathrm{mL}$ M-CSF and $20 \mathrm{ng} / \mathrm{mL}$ RANKL (R\&D Systems, Minneapolis, MN, USA) for 7 days. In a subset of the experiment, $10^{5} /$ well macrophages were incubated in a mixture of osteoclastogenic medium containing $20 \mathrm{ng} / \mathrm{mL}$ TNFSF13b monoclonal antibody, IgG or recombinant TNFSF13b (R\&D Systems, Minneapolis, MN, USA). After incubation, cell cultures were subjected to TRAP cytochemical staining (Sigma-Aldrich Co., St Louis, $\mathrm{MO}$, USA). F-actin ring formation in osteoclasts was probed using F-actin antibody conjugated with Alexa Fluor ${ }^{\circledR} 488$ Phalloidin (Life Technologies, Grand Island, NY, USA) and DAPI-Fluoromount G (Southern Biotech, Birmingham, AL, USA). The number and area of multinuclear cells positive for TRAP stain and fluorescence F-actin rings in 3 fields of each well and 18 wells from 6 animals were counted using a Zeiss inverted microscope and image-analysis software.

\subsection{Pit Formation}

In total, 105 primary bone-marrow macrophages were seeded onto an Osteo Assay Stripwell Plate (Corning, Lowell, MA, USA), which was coated with a bone biomimetic synthetic surface, followed by incubating in osteoclastogenic medium for 7 days, according to the manufacturer's instructions. Culture wells in the absence of primary cells were used as a blank control group. After removal of cell cultures, each well was rinsed with deionized water and then stained by von Kossa staining. Pits that were negative for von Kossa staining on the bone biomimetic synthetic surface were subjected to microscopy [45]. Areas of pits in 3 fields of each well and 18 wells from 6 mice were counted. Percentile pit formation was calculated as areas of pits/areas of microscopic field $\times 100 \%$.

\subsection{Quantitative RT-PCR}

Total RNA in macrophage cultures and bone tissue was isolated using QIAzol reagent (Qiagene, Valencia, CA, USA). Upon reverse transcription of $1 \mu \mathrm{g}$ total RNA, mRNA expression was detected using primers for NFATc1 (forward: 5'-GAAGGTGTACTCCTCGGGTGG-3'; reverse: 5'-GATACCTGGCTCGGTAACACCAC-3'), V-ATPase (forward: 5' ${ }^{\prime}$-AGAAAGCCAAGTGCCTACTCC-3'; reverse: 5'-AAAGGGAAGGGTTTCTTTTGG-3'), MMP9 (forward: 5'-GGGAAGGCTCTGCTGTTCA-3'; reverse: $5^{\prime}$-CGGTTGAAGCAAAGAAGGAG-3'), cathepsin $\mathrm{K}$ (forward: 5'-CCTGCGGCATTACCAACAT-3'; reverse: 5'-GCTGCAGGACTCCAATGTCT-3'), TNFSF13b (forward: $5^{\prime}$-TTCCATGGCTTCTCAGCTTT-3'; reverse: $5^{\prime}$-CGTCCCCAAAGACGTGACT-3'), and actin (forward: 5'-GACGGCCAGGTCATCACTAT-3'; reverse: 5'-CTTCTGCATCCTGTCAGCAA-3'). 
Equation $2^{-\Delta \Delta \mathrm{Ct}}$, where $\Delta \Delta \mathrm{Ct}=\Delta \Delta \mathrm{Ct} \mathrm{t}_{\text {glucocorticoid }}-\Delta \mathrm{C} \mathrm{t}_{\text {vehicle }}$ and $\Delta \Delta \mathrm{Ct}=\mathrm{C} \mathrm{t}_{\text {gene }}-\mathrm{Ct}_{\text {actin }}$ was adopted to calculated the relative expression of each gene.

\subsection{Immunoblotting}

Protein lysates in bone tissue was extracted using PRO-PREPTM Extraction Kits (iNtRON Biotechnology, Sungnam, Korea), according to the manufacturer's instruction. SOCS2 and actin in bone tissue proteins were probed using Western blotting protocols along with SOCS2 and actin antibodies (Cell Signaling Technology, Danvers, MA, USA) and horseradish peroxidase-conjugated IgG that was visualized by chemiluminescence agents.

\subsection{Chromatin Immuneprecipitation (ChIP)-PCR}

Bone tissue extracts were immunoprecipitated with SOCS2 monoclonal antibodies or IgG (Millipore, Billerica, MA, USA). For isolating chromatin, the immunocomplexes were subjected to sonication, elution and Proteinase K digestion using Megan ChIP A/G kits (Millipore, Billerica, MA, USA), according to the manufacturer's instructions. Chromatin was probed by Cy3-labeled primers (forward: 5' -CGTCCTTTGGTCTTGCACTT-3'; reverse: 5'-GGATTGTGGGTTCAG GGTTA-3') for TNFSF13b proximal promoter region (NCBI Accession: NM_033622) using PCR protocols. Enrichment of SOCS2 in designated promoter regions was calculated as \% input as previously described [46].

\subsection{Transfection}

For transfection, $1 \mathrm{nM}$ miR-29a precursor, $1 \mathrm{nM}$ miR-29a antisense oligonucleotide or $1 \mathrm{nM}$ scramble control (Applied Biosystems-Ambio Inc., Austin, TX, USA) were mixed with Lipofectamine 2000 (Invitrogen; Thermo Fisher Scientific, Inc., Waltham, MA, USA), according to the manufacturer's instructions. MC3T3-E1 osteoblasts $\left(5 \times 10^{5}\right.$ cells/well, 6-well plates) were transfected with the mixtures followed by incubating in DMEM with $10 \%$ fetal bovine serum with or without $1 \mu \mathrm{M}$ dexamethasone for $24 \mathrm{~h}$, as previously described [22]. In a subset experiment, cell cultures were transfected with $1 \mu \mathrm{g}$ SOCS2 RNAi and incubated in $1 \mu \mathrm{M}$ dexamethasone for $24 \mathrm{~h}$. Total RNA in cell cultures was isolated for RT-qPCR analysis of miR-29a, SOCS2 and TNFSF13b expression.

\subsection{Statistical Analysis}

Data were expressed as means \pm standard errors. Differences among miR-29a transgenic mice and wild-type mice with glucocorticoid or vehicle treatment were analyzed by a parametric ANOVA test and a Bonferroni post-hoc test. A $p$ value of $<0.05$ was considered statistically significant.

Author Contributions: Study conception and design: R.-W.W., Y.-S.C., W.-S.L., C.-W.K., H.-C.K., C.-K.H., S.-Y.W., J.-Y.K., F.-S.W.; acquisition of data: Y.-S.C., W.-S.L., C.-W.K., H.-C.K., C.-K.H., S.-Y.W.; analysis and interpretation of data: R.-W.W., W.-S.L., J.-Y.K., F.-S.W.; article drafting and revision: R.-W.W., Y.-S.C., W.-S.L., J.-Y.K., F.-S.W.

Finding: This work was supported by a grant [NHRI-EX108-10736SI] from the National Health Research Institute, a grant [MOST-107-2314-B-182A-006-MY3] from the Ministry of Science \& Technology and grants [CMRPG8I0061] from Chang Gung Memorial Hospital, Taiwan.

Acknowledgments: We are grateful to Core Laboratories for Phenomics \& Diagnostics. We appreciate the use of facilities at Chang Gung Memorial Hospital.

Conflicts of Interest: The authors declare no conflict of interest.

\section{References}

1. Buckley, L.; Humphrey, M.B. Glucocorticoid-induced osteoporosis. N. Engl. J. Med. 2018, 379, $2547-2556$. [CrossRef] [PubMed]

2. Hardy, R.S.; Zhou, H.; Seibel, M.J.; Cooper, M.S. Glucocorticoid and bone: Consequences of endogenous and exogenous excess and replacement therapy. Endocr. Rev. 2018, 39, 519-548. [CrossRef] [PubMed] 
3. Rizzoli, R.; Biver, R. Glucocorticoid-induced osteoporosis: Who to treat with what agent. Nat. Rev. Rheumatol. 2015, 11, 98-109. [CrossRef] [PubMed]

4. Bellini, G.; Torella, M.; Manzo, I.; Tortora, C.; Luongo, L.; Punzo, F.; Cloacurci, N.; Nobili, B.; Maione, S.; Rossi, F. PKC $\beta$ II-mediated cross-talk of TRPV1/CB2 modulates the glucocorticoid-induced osteoclast overactivity. Pharmacol. Res. 2017, 115, 267-274. [CrossRef] [PubMed]

5. Fujihara, Y.; Kondo, H.; Noguchi, T.; Togari, A. Glucocorticoids mediate circadian timing in peripheral osteoclasts resulting in the circadian expression rhythm of osteoclast-related genes. Bone 2014, 61, 1-9. [CrossRef]

6. Sabokbar, A.; Mahoney, D.J.; Hemingway, F.; Athanasou, N.A. Non-canonical (RANKL-independent) pathways of osteoclast differentiation and their role in musculoskeletal diseases. Clin. Rev. Allergy Immunol. 2016, 51, 16-26. [CrossRef]

7. Jiang, P.; Gao, W.; Ma, T.; Wang, R.; Piao, Y.; Dong, X.; Wang, P.; Zhang, X.; Liu, Y.; Su, W.; et al. CD137 promotes bone metastasis of breast cancer by enhancing the migration and osteoclast differentiation of monocytes/macrophages. Theranostics 2019, 9, 2950-2966. [CrossRef]

8. Brunetti, G.; Faienza, M.F.; Colaianni, G.; Gigante, I.; Oranger, A.; Pignataro, P.; Ingravallo, G.; Di Benedetto, A.; Bortolotti, S.; Di Comite, M.; et al. Impairment of bone remodeling in LIGHT/TNFSF14-deficient mice. J. Bone Miner. Res. 2018, 33, 704-719. [CrossRef]

9. Hemingway, F.; Taylor, R.; Knowles, H.J.; Athanasou, N.A. RANKL-independent human osteoclast formation with APRIL, BAFF, NGF, IGF-I and IGF-II. Bone 2011, 48, 938-944. [CrossRef]

10. Neri, P.; Kumar, S.; Fulciniti, M.T.; Vallet, S.; Chhetri, S.; Mukherjee, S.; Tai, Y.; Chauhan, D.; Tassone, P.; Venuta, S.; et al. Neutralizing B-cell factor antibody improves survival and inhibits osteoclastogenesis in a severe combined immunodeficient human multiple myeloma model. Clin. Cancer Res. 2007, 13, 5903-5909. [CrossRef]

11. Van Meurs, J.B.; Boer, C.G.; Lopez-Delgado, L.; Riancho, J.A. Role of epigenomics in bone and cartilage disease. J. Bone Miner. Res. 2019, 34, 215-230. [CrossRef] [PubMed]

12. Guo, L.; Chen, K.; Yuan, J.; Huang, P.; Xu, X.; Li, C.; Qian, N.; Qi, J.; Shao, Z.; Deng, L.; et al. Estrogen inhibits osteoclasts formation and bone resorption via microRNA-27a targeting PPAR $\gamma$ and APC. J. Cell Physiol 2018, 234, 581-594. [CrossRef] [PubMed]

13. Xu, R.; Shen, X.; Si, Y.; Fu, Y.; Zhu, W.; Xiao, T.; Fu, Z.; Zhang, P.; Cheng, J.; Jiang, H. MicroRNA-31a-5p from aging BMSCs links bone formation and resorption in the aged bone marrow microenvironment. Aging Cell 2018, 17, e12794. [CrossRef] [PubMed]

14. Nakamachi, Y.; Ohnuma, K.; Uto, K.; Noguchi, Y.; Saegusa, J.; Kawano, S. MicroRNA-124 inhibits the progression of adjuvant-induced arthritis in rats. Ann. Rheum. Dis. 2016, 75, 601-608. [CrossRef] [PubMed]

15. Krzeszinski, J.Y.; Wei, W.; Huynh, H.; Jin, Z.; Wang, X.; Chang, T.C.; Xie, X.J.; He, L.; Mangala, L.S.; Lopez-Berestein, G.; et al. miR-34a blocks osteoporosis and bone metastasis by inhibiting osteoclastogenesis and Tgif2. Nature 2014, 512, 431-435. [CrossRef]

16. Wei, W.; He, H.B.; Zhang, W.Y.; Zhang, H.X.; Bai, J.B.; Liu, H.Z.; Cao, J.H.; Chang, K.C.; Li, X.Y.; Zhao, S.H. miR-29 targets Akts to reduce proliferation and facilitate differentiation of myoblasts in skeletal muscle development. Cell Death Dis. 2013, 4, e668. [CrossRef]

17. Guêrit, D.; Brondello, J.M.; Chuchana, P.; Philipot, D.; Toupet, K.; Bony, C.; Jorgensen, C.; Noël, D. FoxO3a regulation by miRNA-29a controls chondrogenic differentiation of mesenchymal stem cells and cartilage formation. Stem Cells Dev. 2014, 23, 1195-1205. [CrossRef]

18. Parker, M.W.; Rossi, D.; Peterson, M.; Smith, K.; Sikstrom, K.; White, E.S.; Connett, J.E.; Henke, C.A.; Larsson, O.; Bitterman, P.B. Fibrotic extracellular matrix activates a profibrotic positive feedback loop. J. Clin. Invest. 2014, 124, 1622-1635. [CrossRef]

19. Smith, K.M.; Guerau-de-Arellano, M.; Costinean, S.; Williams, J.L.; Bottoni, A.; Mayrikis Cox, G.; Satoskar, A.R.; Croce, C.M.; Racke, M.K.; Lovett-Racke, A.E.; et al. miR-29ab1 deficiency identifies a negative feedback loop controlling Th1 bias that is dysregulated in multiple sclerosis. J. Immunol. 2012, 189, 1567-1576. [CrossRef]

20. Ma, F.; Xu, S.; Liu, X.; Zhang, Q.; Xu, X.; Liu, M.; Hua, M.; Li, N.; Yao, H.; Cao, X. The microRNA miR-29 controls innate and adaptive immune responses to intracellular bacterial infection by targeting interferon- $\gamma$. Nat. Immunol. 2011, 12, 861-869. [CrossRef] 
21. Fukata, T.; Mizushima, T.; Nishimura, J.; Okuzaki, D.; Wu, X.; Hirose, H.; Yokoyama, Y.; Kubota, Y.; Nagata, K.; Tsujimura, N.; et al. The supercarbonate apatite-microRNA complex inhibits dextran sodium sulfate-induced colitis. Mol. Ther. Nucleic Acids 2018, 12, 658-671. [CrossRef] [PubMed]

22. Ko, J.Y.; Chuang, P.C.; Su, W.H.; Ke, H.C.; Chen, Y.S.; Sun, Y.C.; Wang, F.S. MicroRNA-29a mitigates glucocorticoid induction of bone loss and fatty marrow by rescuing Runx2 acetylation. Bone 2015, 81, 80-88. [CrossRef] [PubMed]

23. Søe, K.; Dalaissé, J.M. Glucocorticoids maintain human osteoclasts in the active mode of their resorption cycle. J. Bone Mineral. Res. 2010, 25, 184-192. [CrossRef] [PubMed]

24. Sato, A.Y.; Cregor, M.; McAndrews, K.; Li, T.; Condon, K.W.; Plotkin, L.; Bellido, T. Glucocorticoid-induced bone fragility is prevented in female mice by blocking Pyk2/Anoikis signaling. Endocrinology 2019, 160, 1659-1673. [CrossRef] [PubMed]

25. Conway, H.H.; Henning, P.; Lie, A.; Tuckermann, J.; Lemer, U.H. Activation of dimer glucocorticoid receptors in osteoclast progenitors potentiates RANKL induced mature osteoclast bone resorbing activity. Bone 2016, 93, 43-54. [CrossRef]

26. Lozano, C.; Duroux-Richard, I.; Firat, H.; Schordan, E.; Apparailly, F. MicroRNAs: Key regulators to understand osteoclast differentiation? Front. Immunol. 2019, 10, 375. [CrossRef]

27. Liu, J.; Li, D.; Dang, L.; Liang, C.; Guo, B.; Lu, C.; He, X.; Cheung, H.Y.; He, B.; Liu, B.; et al. Osteoclastic miR-214 targets TRAF3 to contribute to osteolytic bone metastasis of breast cancer. Sci. Rep. 2017, 17, 40487. [CrossRef]

28. Alam, I.; Oakes, D.K.; Reilly AMBillingsley, C.; Sbeta, S.; Gerard-O’Riley, R.L.; Acton, D.; Sato, A.; Bellido, T.; Econs, M.J. Overexpression of WNT16 does not prevent cortical bone loss due to glucocorticoid treatment in mice. JBMR Plus 2018, 3, e10084. [CrossRef]

29. Piemontese, M.; Xiong, J.; Fujiwara, Y.; Thostenson, J.D.; O’Brien, C.A. Cortical bone loss caused by glucocorticoid excess requires RANKL production by osteocytes and is associated with reduced OPG expression in mice. Am. J. Physiol. Endocrinol. Metab. 2016, 311, E587-E593. [CrossRef]

30. Rossi, M.; Pitari, M.R.; Amodio, N.; Di Martino, M.T.; Conforti, F.; Leone, E.; Botta, C.; Paolino, F.M.; Del Giudice, T.; Iuliano, E.; et al. miR-29b negatively regulates human osteoclastic cell differentiation and function: Implications for the treatment of multiple myeloma-related bone disease. J. Cell Physiol. 2013, 228, 1506-1515. [CrossRef]

31. Sul, O.J.; Rajasekaran, M.; Park, H.J.; Suh, J.H.; Choi, H.S. MicroRNA-29b enhances osteoclast survival by targeting BCL-2-modifying factor after lipopolysaccharide stimulation. Oxid. Med. Cell. Longev. 2019, 2019, 1-11. [CrossRef] [PubMed]

32. Franceschetti, T.; Kessler, C.B.; Lee, S.K.; Delany, A.M. miR-29 promotes murine osteoclastogenesis by regulating osteoclast commitment and migration. J. Biol. Chem. 2013, 288, 33347-33360. [CrossRef] [PubMed]

33. Collins, F.L.; Williams, J.O.; Bloom, A.C.; Singh, R.K.; Jordan, L.; Stone, M.D.; McCabe, L.R.; Wang, E.C.Y.; Williams, A.S. CCL3 and MMP-9 are induced by TL1A during death receptor 3 (TNFRSF25)-dependent osteoclast function and systemic bone loss. Bone 2017, 97, 94-104. [CrossRef] [PubMed]

34. Tai, Y.T.; Acharya, C.; An, G.; Moschetta, M.; Zhong, M.Y.; Feng, X.; Cea, M.; Cagnetta, A.; Wen, K.; van Eenennaam, H.; et al. APRIL and BCMA promote human multiple myeloma growth and immunosuppression in the bone marrow microenvironment. Blood 2016, 127, 3225-3236. [CrossRef] [PubMed]

35. Spences, S.; Fitzsimons, A.; Boyd, C.R.; Kessler, J.; Fitzgerald, D.; Elliott, J.; Gabhann, J.N.; Smith, S.; Sica, A.; Hams, E.; et al. Suppression of cytokine signaling 2 and 3 diametrically control macrophage polarization. Immunity 2013, 38, 66-78. [CrossRef]

36. Pass, C.; MacRae, V.E.; Huesa, C.; Faisal Ahmed, S.; Farquharson, C. SOCS2 is the critical regulator GH action in murine growth plate chondrogenesis. J. Bone Miner. Res. 2012, 27, 1055-1066. [CrossRef]

37. Dobie, R.; Ahmed, S.F.; Staines, K.A.; Pass, C.; Jasim, S.; MacRae, V.E.; Farquharson, C. Increased linear bone growth by GH in the absence of SOCS2 is independent of IGF-1. J. Cell Physiol. 2015, 230, 2796-2806. [CrossRef]

38. Moghaddam, A.S.; Afshari, J.T.; Esmaeili, S.A.; Saburi, E.; Joneidi, Z.; Momtazi-Borojeni, A.A. Cardioprotective microRNAs: Lessons from stem cell-derived exosomal microRNAs to treat cardiovascular disease. Atherosclerosis 2019, 285, 1-9. [CrossRef] 
39. Cai, J.; Oiao, B.; Gao, N.; Lin, N.; He, W. Oral squamous cell carcinoma-derived exosomes promote M2 subtype macrophage polarization mediated by exosome-enclosed miR-29a-3p. Am. J. Physiol. Cell Physiol. 2019, 316, C731-C740. [CrossRef]

40. Korabecna, M.; Koutova, L.; Tesarova, P. The potential roles of vesicle-enclosed miRNAs in communication between macrophages and cancer cells in tumor microenvironment. Neoplasma 2017, 64, 406-411. [CrossRef]

41. Asano, J.; Nakano, A.; Oda, A.; Amou, H.; Hiasa, M.; Takeuchi, K.; Miki, H.; Nakamura, S.; Harada, T.; Fuji, S.; et al. The serine/threonine kinase Pim-2 is a novel anti-apoptotic mediator in myeloma cells. Leukemia 2011, 25, 1182-1188. [CrossRef] [PubMed]

42. Kim, H.J.; Zhao, H.; Kitaura, H.; Bhattacharyya, S.; Brewer, J.A.; Muglia, L.J.; Ross, F.P.; Teitelbaum, S.L. Glucocorticoids suppress bone formation via the osteoclast. J. Clin. Invest. 2006, 116, 2152-2160. [CrossRef] [PubMed]

43. Dempster, D.W.; Compston, J.E.; Drezner, M.K.; Glorieux, F.H.; Kanis, J.A.; Malluche, H.; Meunier, P.J.; Ott, S.M.; Recker, R.R.; Parfitt, A.M. Standardized nomenclature, symbols, and units for bone histomorphometry: A 2012 update of the report of the ASBMR Histomorphometry Nomenclature Committee. J. Bone Miner Res. 2013, 28, 1-16. [CrossRef] [PubMed]

44. Moon, Y.M.; Lee, S.Y.; Her, Y.M.; Ryu, J.G.; Kim, E.K.; Son, H.J.; Kwok, S.K.; Ju, J.H.; Yang, C.W.; Park, S.H.; et al. Gene associated with retinoid-interferon-induced mortality 19 attenuates murine autoimmune arthritis by regulation of th17 and treg cells. Arthritis Rheumatol 2014, 66, 569-578. [CrossRef] [PubMed]

45. Ferron, M.; Settembre, C.; Shimazu, J.; Lacombe, J.; Kato, S.; Rawlings, D.J.; Ballabio, A.; Karsenty, G. A RANKL-PKC $\beta$-RFEB signaling cascade is necessary for lysosomal biogenesis in osteoclasts. Gene. Dev. 2013, 27, 955-969. [CrossRef]

46. Lian, W.S.; Ko, J.Y.; Wu, R.W.; Sun, Y.C.; Chen, Y.S.; Wu, S.L.; Weng, L.H.; Jahr, H.; Wang, F.S. MicroRNA-128 repressed chondrocyte autophagy exacerbates knee osteoarthritis by disrupting Atg12. Cell Death Dis. 2018, 9, 919. [CrossRef]

(C) 2019 by the authors. Licensee MDPI, Basel, Switzerland. This article is an open access article distributed under the terms and conditions of the Creative Commons Attribution (CC BY) license (http://creativecommons.org/licenses/by/4.0/). 